\title{
The poor man's capillary microscope. A novel technique for the assessment of capillary morphology
}

\author{
Rupert M Bauersachs, Frank Lößner
}

\begin{abstract}
Objectives-To evaluate a new method for rapid and efficient assessment of capillary morphology.
\end{abstract}

Methods-Nailfold capillary morphology in 18 patients with Raynaud's phenomenon was investigated with a new bedside test, based on a modified dermatoscope using a novel gel-immersion technique. These findings were compared with those obtained by standard capillary microscopy.

Results-With the standard microscope, six patients had dilated capillaries, six had mega-capillaries, seven had avascular fields, eight had ramified capillaries, six had contorted capillaries, and seven patients showed micro-haemorrhages, respectively. The dermatoscope identified exactly the same patients to have the same capillary abnormalities. One hundred and thirty six fingers were available for pairwise comparison: The $\kappa$ statistic was 0.93 for dilated capillaries, 0.97 for mega-capillaries, 0.93 for avascular areas, 0.78 for ramified capillaries, 0.81 for contorted capillaries, and 0.94 for micro-haemorrhages, respectively. The average examination time was 18 (range 8-30) minutes with the standard microscope and 4 (3-8) minutes with the dermatoscope.

Conclusion-A new diagnostic tool for rapid and efficient examination of nailfold capillaries is described for circumstances when a standard microscope is not available. This study shows that the hand held device can be used in clinical routine with sufficient diagnostic efficacy and little expenditure, both timewise and financially.

(Ann Rheum Dis 1997;56:435-437)

Capillary microscopy is a valuable diagnostic tool that can reveal microangiopathy and early diagnostic signs characteristic for scleroderma. ${ }^{1-3}$ In patients with Raynaud's phenomenon inspection of capillaries may uncover a secondary disease process. ${ }^{45}$ As the standard examination is time consuming, capillaroscopy is commonly deferred in clinical routine to an additional appointment. Furthermore, the full set up of a videomicroscope represents a substantial investment for the rheumatologist in private practice or smaller departments. Therefore, capillary assessment with the means of an ophthalmoscope has been advocated. ${ }^{6-8}$
Despite several drawbacks, this method has found some support, mainly because of the lack of other simple and inexpensive methods. Unfortunately, too often capillary morphology is not assessed at all because of the lack of equipment or time.

Therefore, there is a need for a quick and simple capillary investigation technique. This study evaluates whether a modified dermatoscope with a new immersion technique can be used as a diagnostic tool for the examination of nailfold capillaries, and how its findings compares with those obtained with a standard capillary microscope.

\section{Methods}

We modified a single lens dermatoscope (epiluminescence microscope: Heine Dermatoskop Delta 10, Heine Optotechnik, 82211 Herrsching, Germany (fig 1), which initially had been developed for the inspection of skin lesions and malignant melanoma. ${ }^{10}$ For the examination of nailfold capillaries several adaptations had to be made: (1) The single lens magnification was changed from $10 \times$ to $12 \times$; (2) the light bulb was tinted green (wavelength approximately $490 \mathrm{~nm}$ ) to increase the contrast of the red cell column in the capillaries (bulb paint by Conrad Electronic, Hirschau, Germany); (3) the original bulbs were replaced by $2.5 \mathrm{~V}$ Kryptogen bulbs (Heine Optotechnik, 82211 Herrsching, Germany); (4) a graded (millimeter) contact slide was used on the covering surface of the device to estimate the size of avascular areas and capillary diameter; the thickness of the lines was 50 and $100 \mu \mathrm{m}$, respectively; (5) common immersion oil was substituted by a Carbachol gel (ultrasound transmission gel, General Pharmacy, Hospital Bogenhausen, Munich, Germany), which can easily be applied between the fingernail and the dermatoscope without running off the finger during the examination. We measured a refractional index of 1.348 of the gel.

The examiner using the dermatoscope had no knowledge of the videomicroscope findings and vice versa. The reference microscope was a stereoscopic, epiluminescence microscope (WILD M3B, Heerbrugg, Switzerland), with video recording and analysis (Panasonic AG-6400-E, Matsushita Electric Industrial Co, Ltd, Japan; TM-150 PSN Monitor, JVC, Yokohama, Japan). The magnification was 60-fold to 240-fold; the examination followed a standard procedure. ${ }^{11}$ For the dermatoscope examination, a small portion of immersion gel 


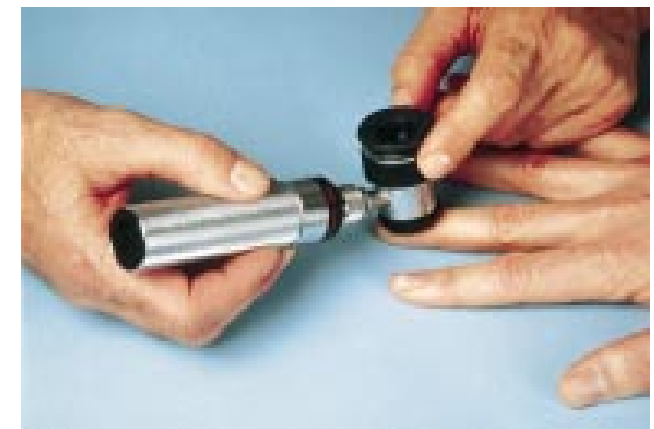

Figure 1 Dermatoscope with modifications made for capillary examination, using ultrasound transmission gel for immersion.

was placed onto the nailfold, and the dermatoscope was placed into direct contact with gel and the finger, so that the glass was completely immersed in the gel. Usually, the whole nailfold was envisioned within one or two eyefields. Qualitative assessment of the following parameters was performed for both the dermatoscope and the standard microscope: Visibility (sufficient, poor), dilated capillaries (diameter $>20 \mu \mathrm{m}$ ), mega-capillaries (diameter $>50 \mu \mathrm{m})$, avascular areas $(>0.5 \mathrm{~mm})$, ramified capillaries, contorted capillaries, and micro-haemorrhages. The average examination time for one patient (eight fingers excluding digiti I) with the standard microscope without photo or video documentation was 18 (range 8-30) minutes, with the dermatoscope 4 (range $3-8$ ) minutes. The $\kappa$ test statistic was used for statistical analysis of the correlation between the findings of the two methods. ${ }^{72}$

PATIENTS

We examined 18 patients (14 women, four men) who were referred for a diagnostic examination because of Raynaud's phenomenon. Discharge diagnoses were: seven patients with scleroderma, three with systemic lupus erythematosus, two with rheumatoid arthritis, one with Sharp's syndrome; five patients had Raynaud's phenomenon without an additional diagnosis.

\section{Results}

Using the standard microscope, patients with the following capillary abnormalities were identified: six patients had dilated capillaries

Table 1 Capillary morphology of individual fingers in 18 patients with Raynaud's phenomenon, assessed with a standard capillary microscope in comparison with the modified dermatoscope

\begin{tabular}{llccl} 
& & \multicolumn{2}{c}{ Dermatoscope } & \\
\cline { 3 - 4 } & Microscope & Present & Absent & \multirow{2}{*}{$\kappa$} \\
\hline Dilatation & Present & 37 & 3 & 0.93 \\
Mega-capillaries & Absent & 2 & 92 & \\
Avascular fields & Present & 27 & 2 & 0.97 \\
& Absent & 0 & 104 & \multirow{2}{*}{0.93} \\
Ramified capillaries & Present & 27 & 4 & \\
Contorted capillaries & Absent & 1 & 103 & 0.78 \\
Micro-haemorrent & Absent & 2 & 13 & \multirow{2}{*}{0.81} \\
& Present & 100 & 88 & \\
& Absent & 1 & 12 & 0.94 \\
& Present & 35 & 4 &
\end{tabular}

Because of the limited visibility in some fingers the total number of comparisons may differ. The kappa test statistic $(\kappa)$ is used for statistical analysis of the correlation between the two methods. and six had mega-capillaries, in seven patients avascular fields were observed, in eight patients ramified capillaries, and in six contorted capillaries, while micro-haemorrhages were found in seven patients. With the dermatoscope exactly the same patients were identified to have the same capillary abnormalities.

For the assessment of capillary morphology of individual fingers, visibility in a total of 144 fingers was considered sufficient in 126 with the videomicroscope and in 124 fingers with the dermatoscope, respectively. Table 1 shows the results of the pairwise comparison of the two methods for individual fingers. Note, that because of impaired visibility in some fingers, on average only 136 pairs were usable for comparison.

\section{Discussion}

We present a new diagnostic tool for quick and efficient examination of nailfold capillaries for circumstances when standard microscope equipment is not available. The method is based on a novel immersion technique, which uses a highly viscous immersion gel. This study shows that the hand held device can be used with sufficient diagnostic efficacy in clinical routine with little expenditure, both timewise and financially, as the dermatoscope costs less than a standard ophthalmoscope. The dermatoscope seems to be comparable, if not superior to the ophthalmoscope as judged by data from an earlier report ${ }^{7}$ using the same statistical approach ( $\kappa$ statistic 0.816 to 0.929 for the ophthalmoscope compared with a videomicroscope), even though this would have to be confirmed by direct comparison. For ramified and contorted capillaries there were about $10 \%$ false negative examinations, which illustrates the limits of this method. Still, the practical advantages, the good and straightforward visualisation of capillaries, and the wide eyefield with the dermatoscope is obvious from the very first clinical use. The lack of photographic documentation does not seem to be an important problem, as pathological findings should lead to an extended examination with a standard microscope. The standard $10 \times$ magnification of the dermatoscope may also yield sufficient results, however, it seems that capillary morphology can more readily be assessed with the higher $12 \times$ magnification, even though the depth of focus is more limited.

The dermatoscope, with or without modification, may serve not only the poor man as a simple capillary microscope, but also the rich man, who may not have the time to use his more elaborate apparatus whenever indicated.

This study was supported by a grant from the Heinrich und Fritz Riese Stiftung.

1 Brown GE, O'Leary PA. Skin capillaries in scleroderma. Arch Intern Med 1926;36:73-88.

2 Maricq HR, LeRoy EC. Patterns of finger capillary abnormalities in connective tissue disease by "widefield" microscopy. Arthritis Rheum 1973;16:619-28.

3 Maricq HR, LeRoy EC, D'Angelo WA, Medsger TAJ, Rodnan GP, Sharp GC, et al. Diagnostic potential of in vivo capillary microscopy in scleroderma and related disorders. capilary microscopy in scleroder

4 Blockmans D, Vermylen J, Bobbaers H. Nailfold capillaroscopy in connective tissue disorders and in Raynaud's phenomenon. Acta Clin Belg 1993;48:30-41. 
5 Zufferey P, Depairon M, Chamot AM, Monti M. Prognostic significance of nailfold capillary microscopy in patients with Raynaud's phenomenon and scleroderma-pattern abnormalities. A six-year follow-up study. Clin Rheumato 1992;11:536-41.

6 Herd JK. Nailfold capillary microscopy made easy. [Letter] Arthritis Rheum 1976; 19:1370-1.

7 Ranft J, Lammersen T, Heidrich H. In vivo capillary microscopy findings and ophthalmoscopy findings in scleroderma. Arthritis Rheum 1987;30:1173-5.

8 Minkin W, Rabhan NB. Office nailfold capillary microscopy using the ophthalmoscope. J Am Acad Dermatol 1982; $7: 190-3$
9 Braun-Falco O, Stolz W, Bilek P, Merkle T, Landthaler M. Das Dermatoskop. Eine Vereinfachung der Auflichtmikroskopie von pigmentierten Hautveränderungen. Hautarzt 1990; 41:131-5.

$10 \mathrm{Kerl} \mathrm{H}$, Wolf IH, Sterry W, Soyer HP. Dermatoskopie. Eine neue Methode zur klinischen Diagnose des malignen Melanoms. Dtsch Med Wochenschr 1995;120:801-5.

11 Bollinger A, Fagrell B. Techniques and normal findings. In: Bollinger A, Fagrell B, eds. Clinical capillaroscopy. Toronto: Hofgrefe and Huber, 1982:1-8.

12 Fleiss JL. Measuring nominal scale agreement among many raters. Psychol Bull 1971; 76:378-82.
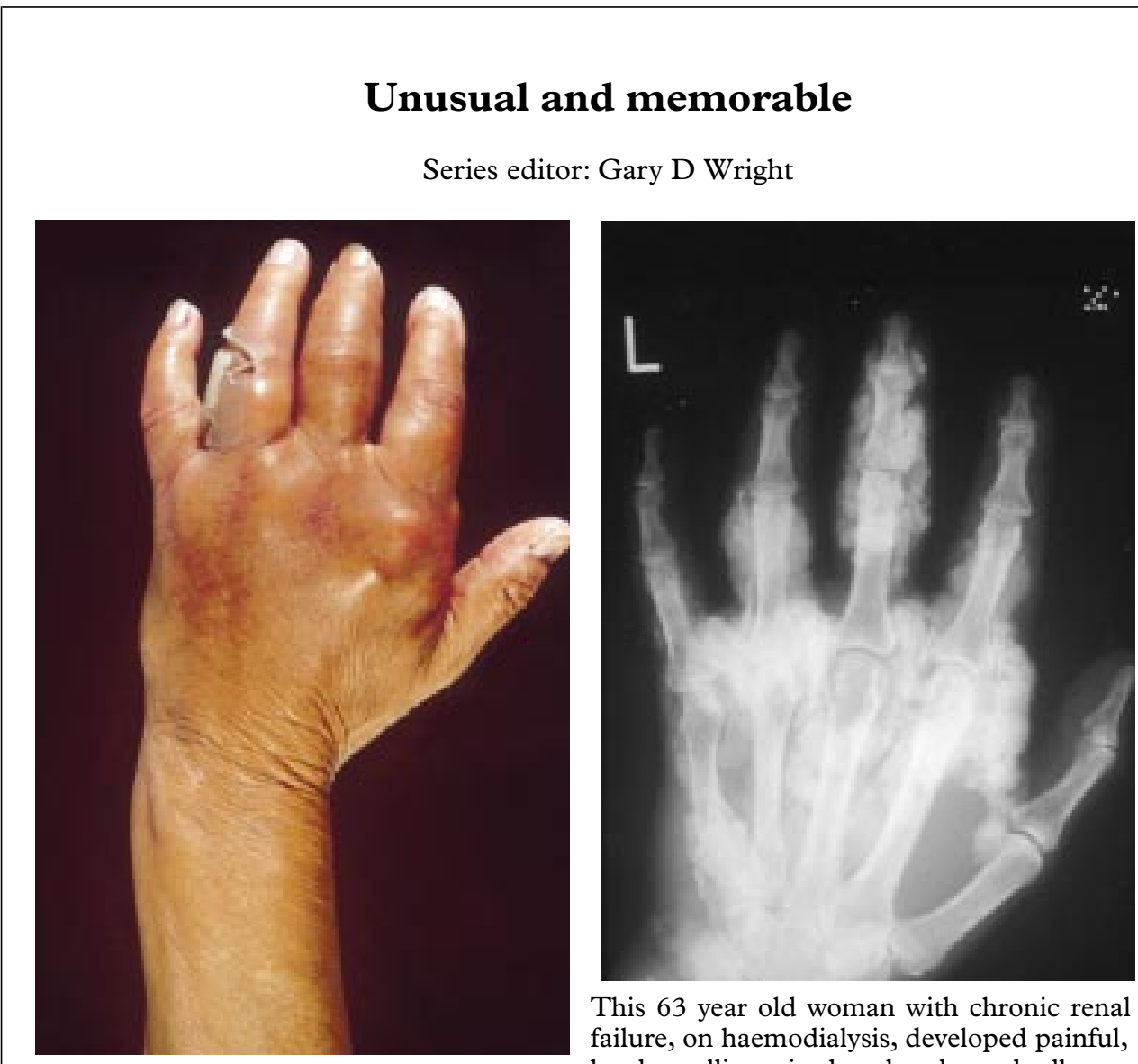

This 63 year old woman with chronic renal failure, on haemodialysis, developed painful, hard swellings in her hands and elbows. Radiographs confirmed calcinosis, and material aspirated from a finger was confirmed as hydroxyapatite.

Soft tissue calcification in chronic renal failure may be visceral or non-visceral. Various calcium crystals may be responsible, most commonly calcium phosphates and oxalates. ${ }^{1}$ Visceral calcification, in heart, lung and muscle, is usually an amorphous compound similar to whitlockite (anhydrous calcium carbonate). Non-visceral calcification in arteries, skin, and periarticular tissues is more commonly hydroxyapatite. ${ }^{2}$ Deposits may undergo a fibrotic reaction and become encapsulated, appear multiloculated, and spontaneously discharge through the skin.

1 Reginato AJ, Kurnik B. Calcium oxalate and other crystals associated with kidney diseases and arthritis. Semin Arthritis Rheum 1989;3:198-224

2 Contiguglia SR, Alfrey AC, Millar NL, Runnells DE, Le Geros RZ. Nature of soft tissue calcification in uremia. Kidney Int 1973;4:229-35.

Contributors: Gary D Wright, Michael DOHERTY. City Hospital, Nottingham, NG5 1PB, United Kingdom. 